\title{
GENETICS AND PERIODONTIUM: A REVIEW
}

\author{
Dr. Baljeet Singh ${ }^{1}$, Dr. Avnika Garg ${ }^{2}$, Dr. Nandini Nayyar ${ }^{3}$, Dr. Alka Sharma ${ }^{4}$ \\ ${ }^{1}$ Prof. \& Head, Department of Periodontics, Bhojia Dental College and Hospital, Distt. Solan, Himachal Pradesh (India) \\ ${ }^{2}$ PG Student, Department of Periodontics, Bhojia Dental College and Hospital, Distt. Solan, Himachal Pradesh (India) \\ ${ }^{3}$ Sr. Lecturer, Department of Periodontics, Bhojia Dental College and Hospital, Distt. Solan, Himachal Pradesh (India) \\ ${ }^{4}$ PG Student Department of Periodontics, Bhojia Dental College and Hospital, Distt. Solan, Himachal Pradesh (India) \\ Corresponding Author: \\ ${ }^{1}$ Mobile: 919814510711 Email: baljeet065_singh@yahoo.co.in
}

\begin{abstract}
Received :

$10^{\text {th }}$ April, 2013

Accepted:

$13^{\text {th }}$ June, 2013

Available online:

$25^{\text {th }}$ August, 2013

Periodontal disease may be regarded as a range of different diseases for which certain individuals are at relatively high risk. Epidemiological and molecular studies of the oral microbial flora suggest, that although microbial factors are required for periodontal disease, they alone do not predict the presence or severity of periodontitis. So in high-risk patient groups, host factors appear to play an important role in susceptibility to periodontitis. In recent years elements of host susceptibility, such as immune response and systemic disease state, and other non-microbial environmental factors, such as smoking, have been shown to be important contributors to the disease expression. Thus, periodontitis represents a lifelong account of interactions between our genome, our behavior, and our environment.

Keywords: Genetics, Periodontium, Periodontitis.
\end{abstract}

\section{INTRODUCTION}

Periodontitis is a chronic infectious disease of the supporting tissues of the teeth. Due to bacterial infection, periodontal tissues become inflamed, and are slowly destroyed by the action of the inflammatory process. If the disease is left untreated, teeth lose their ligamentous support to the alveolar bone, alveolar bone itself is resorbed, and the teeth become mobile and are finally lost. ${ }^{1}$ Traditionally, periodontitis was thought to be strictly environmental in origin. Despite this belief, it was recognized that only a portion of the variability of disease in the population could be explained by environmental factors alone. In a classic study of the natural history of periodontitis, Loe etal found that among individuals with poor oral hygiene and no access to dental care, some developed disease at a rapid rate, whereas others experienced little or no disease. This variation must have been attributable to either unrecognized environmental factors or to individual differences in susceptibility to disease. Because host susceptibility may be defined in terms of genetic variation, a relatively recent focus in periodontology has been to quantify genetic risk and identify specific gene variants that determine disease susceptibility. At present, the specific role that genes play in defining susceptibility remains largely unknown. ${ }^{2}$

Moreover, periodontitis is considered to be a complex disease. Common features of complex human diseases (for example Alzheimer's disease, Crohn's disease and cardiovascular diseases) are that they present mostly a relatively mild phenotype, are slowly progressive and chronic in nature. ${ }^{3}$ The pathophysiology of complex diseases is characterized by various biological pathways, leading to similar clinical phenomena. Importantly, complex diseases are associated with variations in multiple genes, each of which has a small overall contribution and relative risk for the disease process. ${ }^{4}$ Similarly to other complex diseases, we estimate that, for periodontitis, at least 10 and possibly as many as 20 diseasemodifying genes may be involved. However, it is important to realize that the number and types of disease-modifying genes for the same condition may not be equal for different forms of periodontitis and different ethnic populations; their effect is also influenced by environmental factors (geneenvironment interactions). ${ }^{1}$ 


\section{GENETIC SUSCEPTIBILITY TO PERIODONTITIS}

In periodontics, as well as in medicine, we are interested in the genetics of both humans and pathogens and the genetic interaction between them. Of importance is the increasing awareness that most of the destructive processes involved in periodontitis are host-derived. These inflammatory processes leading to destruction of periodontal tissues as well as the properties that result in their regeneration and the responses to implanted material are of great interest to both researchers and clinicians alike. The information contained within the human genome can potentially lead to a better understanding of these control mechanisms modulating the production of inflammatory mediators as well as provide potential therapeutic targets for periodontal regeneration. A gene interacts directly or through its protein product with many other genes and gene products in coordinated networks, often resulting in striking variations in symptoms among patients with the same disease. ${ }^{5}$ The search for periodontitis-susceptibility alleles is complicated because: (1) Multiple causes (both genetic and non-genetic) may exist for the same disease (etiologic heterogeneity) and (2) Different genetic mechanisms may lead to the same clinical end point (genetic heterogeneity). ${ }^{2}$

\section{GENETIC DISORDERS ASSOCIATED WITH GINGIVAE AND PERIODONTIUM}

The disorders have been grouped according to the resultant protein or biochemical defect(s); the mutant alleles may affect the function of phagocytic immune cells or the structure of epithelia, connective tissue, or the teeth themselves. The specific gene or tissue defect responsible for the condition has been identified for some but not all disorders. ${ }^{6}$

Hereditary gingival fibromatosis type 1: An autosomal dominant form of gingival overgrowth, was found to be associated with a mutation in the SOS (Son of sevenless-1) gene. The insertion mutation introduces a frame-shift creating a truncated protein that abolishes a vital portion of the protein. ${ }^{5}$

Disorders of connective tissue metabolism: Any process which alters the connective tissue matrices, their resident cells, or both would be expected to impact significantly on the tissues of the periodontium. As such, genetic disorders such as the mucopolysaccharidoses, which affect glycosaminoglycans metabolism, mannosidosis, which affects the metabolism of glycoproteins containing mannose and Ehlers-Danlos syndrome (autosomal dominant manner), which affects collagen synthesis may be associated with a variety of periodontal defects.

Skin disorders: Numerous skin disorders have been noted to have a periodontal component. For example, Papillon- Lefevre syndrome (PLS) is a rare autosomal recessive disorder which is characterized by hyperkeratosis of the palms of the hands and soles of the feet, and aggressive periodontitis, typically occurring in childhood. In this condition, the degree of periodontal destruction is so great that the deciduous teeth are exfoliated prematurely and the permanent dentition often follows a similar fate. ${ }^{6}$ PLS is caused by mutations in the cathepsin $\mathrm{C}$ gene, which is located on chromosome 11 (11q14- q21). Cathepsin $\mathrm{C}$ appears to play a role in degrading proteins and activating proenzymes in immune and inflammatory cells. ${ }^{2}$

Metabolic disorders: Another autosomal recessive disease, acatalasia, is characterized by a failure to produce catalase and hence break down hydrogen peroxide, which is a by-product of normal cell activity. The hydrogen peroxide is itself toxic and also produces superoxide radicals leading to tissue destruction. This can be particularly notable in the periodontal tissues, where severe gingival necrosis and marked bone loss has been noted in this condition. $^{7}$ Hypophosphatasia is a rare disorder caused by mutations in the tissue non-specific alkaline phosphatase gene (lp36.1-p34) leading to alterations in bone metabolism. The teeth may be poorly formed, and there is an associated cementum hypoplasia and early periodontal disease with bone loss and tooth loss. The condition leads to the premature loss of the primary teeth and occasionally the permanent teeth. ${ }^{8}$

Disorders of leukocyte function: In most forms of periodontal disease the response of leukocytes within the periodontal milieu is central to the outcome of the disease process. Therefore, any process that leads to diminished numbers of leukocytes or decreased leukocyte function can lead to severe periodontal breakdown. The neutropenias are a family of disorders (agranulocytosis, cyclic neutropenia, familial neutropenia and chronic idiopathic 
neutropenias) of which severe periodontal disease is a common feature. Chediak-Higashi syndrome is a genetically transmitted condition characterized by abnormal neutrophil function. It is inherited as autosomal recessive trait associated with severe early-onset periodontitis. A deficiency in leukocyte adhesion (LAD) has been associated with a defect in the Mac-1 gene (LAD-I) or an absence of the membrane glycoprotein CD15s (LAD11) and appears to have a strong pattern of familial distribution. These defects lead to impaired leukocyte adhesion and are associated with severe forms of early onset periodontitis. ${ }^{6}$

Chromosomal abnormalities: Trisomy 21 (Down syndrome) is one of the best characterized chromosomal defects in which there are associated periodontal problems. In these patients, both earlyonset forms of periodontitis as well as advanced adult type chronic inflammatory periodontal disease have been noted. ${ }^{9}$ Although these individuals are likely to have poor oral hygiene compared with unaffected individuals, a genetic contribution to the periodontal condition has been indicated. One of the most likely contributory factors to the increased level of periodontitis in these individuals is related to impaired neutrophil function. ${ }^{10}$

\section{GENE POLYMORPHISMS IN PERIODONTAL HEALTH AND \\ DISEASE}

Gene polymorphisms are locations within the genome that vary in sequence between individuals and are very prevalent, affecting at least $1 \%$ of the population. Polymorphisms of human genes occur at one or more of the following sites: (1) The promoter or 5'-flanking region; (2) The exon or the gene coding regions; (3) The intron(s) or the gene intervening regions; (4) The 3'-untranslated region. ${ }^{11}$ The most common form of polymorphisms is the single nucleotide polymorphism, which is a change in a single base pair in the genomic DNA. Another class of polymorphism is the simple sequence repeats, of which the common forms are the dinucleotide and trinucleotide repeats. ${ }^{12}$ A third category of gene polymorphism involves insertions or deletions, which can be as small as 1 base. ${ }^{13}$

\section{CYTOKINE GENE} POLYMORPHISMS

A. IL-1 Gene Polymorphisms: Many investigators have reported a positive association between periodontitis and the presence of specific polymorphism of the IL1 gene. However, an unveiled interaction exists between the IL-1 genetic polymorphism and environmental factors such as smoking. Smokers bearing the genotype-positive IL-1 allele combination may be at an increased risk of developing periodontitis. $^{14}$

B. Tumor Necrosis Factor- $\alpha$ (TNF- $\alpha$ ) Gene Polymorphisms: TNF- $\alpha$ is one of the most widely studied cytokines in periodontitis. ${ }^{18}$ Polymorphisms in the promoter region of the TNF- $\alpha$ gene at positions -238 ( $\mathrm{G}$ to $\mathrm{A})$ and -308 ( $\mathrm{G}$ to $\mathrm{A})$ have been reported. The -308 A allele has been associated with high promoter activity and enhanced TNF- $\alpha$ production. ${ }^{15}$

C. IL-10 Gene Polymorphism: IL-I0 is located on chromosome 1 , in a cluster with closely related interleukin genes, including IL-19, IL-20 and IL-24. IL-I0 plays a role in the regulation of pro-inflammatory cytokines such as IL-1 and TNF- $\alpha$. IL-I0 gene polymorphisms have been investigated in relation to aggressive periodontitis. ${ }^{16}$

2. HLA GENETICS: The human leukocyte antigen (HLA) complex plays an important role in immune responsiveness and may be involved in antigen recognition of periodontal pathogens. HLA class II molecules have been identified on immune cells. These molecules are involved in the interaction between $\mathrm{T}$ and $\mathrm{B}$ lymphocytes and in the production of high affinity IgG antibodies. Several investigators have studied populations of patients with different forms of periodontitis to investigate the expression of various HLA antigens. They have studied polymorphisms of HLA-DR molecules in patients with periodontitis and found a significant association between several DRB1 alleles and the disease. ${ }^{15}$

\section{IMMUNO-RECEPTORS (FC $\gamma$ R GENE) POLYMORPHISMS:}

The association of immuno-receptors to periodontitis has been well studied. In particular, 
receptors for the $\mathrm{Fc}$ domain of $\mathrm{IgG}$ ( $\mathrm{Fc}$ gamma R, $\mathrm{FC}_{\Upsilon} \mathrm{R}$ ) provide a critical link between specific humoral responses and the cellular branch of the immune system. ${ }^{17} \mathrm{FC}_{\gamma} \mathrm{Rs}$ are categorized as a family of receptors, expressed on the cell surface of leukocytes, which bind $\mathrm{IgG}$ antibodies and immune complexes. In humans, $\mathrm{FC}_{Y} \mathrm{Rs}$ are expressed on natural killer cells, macrophages, $\mathrm{T}$ lymphocytes, monocytes, and mast cells. The interaction between $\mathrm{FC}_{\mathrm{Y}} \mathrm{Rs}$ and $\mathrm{IgG}$ triggers a variety of biological responses, including phagocytosis, endocytosis, antibody-dependent cellular cytotoxicity, release of inflammatory mediators, and enhancement of antigen presentation. Majority of reports indicate that polymorphisms of $\mathrm{FC}_{\curlyvee} \mathrm{R}$ gene tend to be associated with both aggressive and chronic forms of periodontitis. These alleles may be in linkage disequilibrium with a gene causing periodontitis, although it still remains unclear whether the outcome of periodontitis is associated with the functional defect of $\mathrm{FC}_{\gamma} \mathrm{Rs}^{18}$

\section{MATRIXMETALLOPROTEINASE}

POLYMORPHISMS:Matrix metalloproteinases are one of the most important groups of enzymes involved in periodontal connective tissue destruction. Despite this, there are very few reports concerning polymorphisms of genes for matrix metalloproteinases and periodontitis. Due to the limited number of studies carried out to date, it is difficult to relate single nucleotide polymorphisms of matrix metalloproteinase genes with periodontitis. ${ }^{19}$

\section{STRUCTURAL MOLECULES (CATHEPSIN}

C): Aggressive periodontitis in prepubertal children is often associated with genetic disorders such as Papillon-Lefevre syndrome. This syndrome is associated with mutations in the cathepsin $\mathrm{C}$ gene. Whether the pathogenetic role of cathepsin $\mathrm{C}$ gene variants also relates to types of periodontitis other than syndrome-associated periodontitis remains to be confirmed. ${ }^{15}$

\section{GENE POLYMORPHISMS IN CHRONIC (ADULT) PERIODONTITIS}

A study by Holla etal examined a series of polymorphisms in genes contained within the human luekocyte antigen (HLA) Class III region, including genes for TNF- $\beta$, angiotensin-converting enzyme, and endothelin-1, and found that various combinations of genotypes were associated with chronic periodontitis. It is noteworthy that many investigators have also studied relationships between periodontitis and HLA Class I and Class II antigens. However, definitive relationships have not been found to date, although several associations have been suggested. ${ }^{20} \mathrm{~A}$ polymorphism in the gene coding for an Fc receptor (CD16), FcyRIIIb, was shown to be associated with increased disease recurrence in a Japanese patient cohort. This polymorphism affects the function of polymorphonuclear neutrophils and influences their ability to utilize antibodies efficiently as opsonins; thus, this polymorphism could play a role in the patients' ability to deal with bacterial infections. Additionally, a polymorphism in the $\mathrm{N}$-acetyl transferase gene (NAT-2) has been demonstrated to have an association with periodontitis risk. This polymorphism codes for the 'slow acetylator' phenotype, which is known to be a risk factor for other smoking-related diseases, in particular, cancer susceptibility. ${ }^{21}$

\section{GENE POLYMORPHISMS IN AGGRESSIVE (EARLY ONSET) PERIODONTITIS}

Analogous to chronic periodontitis, polymorphisms in the IL-1 gene complex have been found to be associated with aggressive periodontitis. However, the alternative alleles to those imparting risk for chronic periodontitis were found to be associated with aggressive periodontitis. Also, the FcyRIIIb polymorphism in the Japanese population was found to be associated with risk for generalized aggressive (early onset) periodontitis. ${ }^{22}$ Polymorphisms in the promoter and intron regions of the gene coding for the cytokine IL- 4 have been reported to be associated with early onset periodontitis. This cytokine is important in stimulating production of B lymphocytes and the consequent production of immunoglobulin $\mathrm{G}(\mathrm{IgG})$ and $\mathrm{IgE}$ antibodies, as well as in the differentiation of $\mathrm{T}$ cells. It also inhibits macrophage inflammatory responses such as IL-1 production. Polymorphisms present in the vitamin D receptor (VDR) gene, which have been correlated with both bone mineral density and bone turnover rate, were reported to be associated with localized aggressive periodontitis (but not generalized aggressive periodontitis). ${ }^{23}$ Gwinn etal reported a single nucleotide polymorphism in the gene coding for the IMLP receptor in localized aggressive periodontitis patients. This polymorphism, located in 
the ligand-binding region of this receptor, could explain the defective chemotaxis observed in many such patients. Finally, in 1986, Boughman et al reported that a major gene located on chromosome $4 \mathrm{q}$ was responsible for autosomal dominant transmission of localized aggressive periodontitis in an extended family that also exhibited dentinogenesis imperfect. ${ }^{24}$

\section{FUTURE OF GENETIC STUDIES IN PERIODONTOLOGY}

Prognostic tests: Clinicians tend to view the application of prognostic knowledge in the context of currently available therapeutic approaches, that is, they are accustomed to treating periodontal disease after it has occurred and try to predict whether there will be recurrent disease in the future. Or on the other hand, he could predict the likelihood of disease initiation before it occurs and ultimately will be linked to knowledge of the environmental factors that can initiate the inappropriate activation of genes that are problematic. Such tests would also be useful if they could be used to predict the ultimate success of a variety of therapies.

Diagnostic tests: The currently immutable genetic susceptibility profile for an individual can be used to develop assays of the patient's variable gene expression profile at any given time. This would require identification of the baseline 'activity' of susceptibility genes in subjects with no disease and the change in activity that occurs during and as a result of initiation or progression of disease. It is conceivable that, should such tests be administered at an opportune time, subclinical disease could be detected and appropriate environmental or genetic therapies could be administered.

Gene therapy: It is a technology of introducing foreign genetic material into a patient for correcting its genomic defect. Genetic surgeons can now go deep into the cells and fix those defective genes with a new scalpel-a virus. The inherent benefit of this therapy is to permanently cure the physiological dysfunction by repairing the genetic defect. ${ }^{25}$

\section{CONCLUSION}

Periodontitis is clearly multifactorial, and researchers need to design studies that examine the role of important environmental and genetic factors simultaneously. Given the large numbers of genes in the human genome and bacteria in the oral cavity, it is likely that genes and the environment interact in important but as-yet unrecognized ways to alter disease risk. Most importantly, identifying specific genetic risk factors may be academically appealing but is of little use unless it leads to improvements in the prevention or treatment of disease.

\section{REFERENCES}

1. Marja L. Laine, Wim Crielaard, Bruno G. Loos. Genetic susceptibility to periodontitis. Perio 20002012 February; 58:37-68

2. Newman MG, Takei HH, Klokkevold, Carranza FA. Carranza's Clinical Periodontology. $10^{\text {th }}$ edition. St. Louis 2006; Saunders:193-208.

3. Taylor PC, Feldmann M. New approaches to therapeutic immunomodulation for immune-mediated inflammatory disorders. Curr Opin Pharmacol 2004;4:368-371.

4. Hart TC, Marazita ML, Wright JT. The impact of molecular genetics on oral health paradigms. Crit Rev Oral Bio Med 2000;1:26-56.

5. Salvador Nares. The genetic relationship to periodontal disease. Perio 20002003 June;32:36-49.

6. Michael J. Aldred, P. Mark Bartold. Genetic disorders of the gingiva and periodontium. Perio 20001998 October;18:720.

7. Delgado WA, Calderon R. Acatalasia in two Peruvian siblings. Oral Surg Oral Med Oral Pathol Oral Radiol Endod 1979; 8: 358-368.

8. Whyte MP. Hypophosphatasia. In Scriver CR, Beaudet AL, Sly WS, Valle D,ed. The metabolic and molecular basis of inherited diseases. $7^{\text {th }}$ Edition. New York; McGraw Hills, 1995: 4095.

9. Raeland Bosma W, Van Dijk LJ. Periodontal disease in Down's syndrome: a review. J Clin Periodontol 1986;13: 64-73.

10. Izumi Y, Sugiyama S, Shinozuka O, Yamazaki T, Ohyama $\mathrm{T}$ et al. Defective neutrophil chemotaxis in Down Syndrome patients and its relationship to periodontal destruction. J Periodontol 1989;60:238-242.

11. Jaber BL, Liangos O, Pereira BJ, Balakrishnan VS. Polymorphism of immunomodulatory cytokine genes: implications in acute renal failure. Blood Purif 2004;22: 101-111.

12. Jasinska A, Michlewski G, de Mezer M, Sobczak K, Kozlowski $\mathrm{P}$ et al. Structures of trinucleotide repeats in human transcripts and their functional implications. Nucl Acids Res 2003;31:5463-5468.

13. Reich DE, Lander ES. On the allelic spectrum of human disease. Trend Genet 2001:17:502-510.

14. Meisel P, Schwahn C, Gesch D, Bernhardt O, John U et al. Dose-effect relation of smoking and the interleukin-1 gene polymorphism in periodontal disease. J Periodontol 2004; 75:236-242.

15. Shogo Takashiba and Koji Naruishi. Gene polymorphisms in periodontal health and disease. Perio 20002006 February; 40:94-106.

16. Jan Lindhe. Clinical Periodontolgy and Implant Dentistry. $4^{\text {th }}$ edition. UK; Blackwell Munksgaard:2003. 
17. Meisel P, Carlsson LE, Sawaf H, Fanghaenel J, Greinacher A et al. Polymorphisms of Fc gamma-receptors RIIa, RIIIa, and RIIIb in patients with adult periodontal diseases. Genes Immun 2001;2:258-262.

18. Kinane DF, Hart T. Genes and gene polymorphisms associated with periodontal disease. Crit Rev Oral Biol Med 2003;14:430-449.

19. Holla LI, Fassmann A, Vasku A, Goldbergova M, Beranek M, Znojil V, Vanek J, Vacha J. Genetic variations in the human gelatinase a (matrix metalloproteinase-2) promoter are not associated with susceptibility to, and severity of, chronic periodontitis. J Periodontol 2005;76:1056-1060.

20. Holla LI, Fassmann A, Vasku A, Znojil V, Vanek J, Vacha J. Interactions of lymphotoxin alpha (TNF-beta), angiotensin-converting enzyme (ACE), and endothelin-1 (ET-1) gene polymorphisms in adult periodontitis. J Periodontol 2001;72:85-89.
21. Kobayashi T, Westerdaal NA, Miyazaki A, van der Pol WL, Suzuki T et al. Relevance of immunoglobulin G Fc receptor polymorphism to recurrence of adult periodontitis in Japanese patients. Infect Immun 1997;65:3556-3560.

22. Kobayashi T, Sugita N, van der Pol WL, Nunokawa Y, Westerdaal NA et al. The Fc gamma receptor genotype as a risk factor for generalized early-onset periodontitis in Japanese patients. J Periodontol 2000;71:1425-1432.

23. Hennig BJ, Parkhill JM, Chapple IL, Heasman PA, Taylor JJ. Association of a vitamin D receptor gene polymorphism with localized early-onset periodontal diseases. J Periodontol 1999;70:1032-1038.

24. Gwinn MR, Sharma A, De Nardin E. Single nucleotide polymorphisms of the $\mathrm{N}$-formyl peptide receptor in localized juvenile periodontitis. J Periodontol 1999;70:1194-1201.

25. Harvey A. Schenkein. Finding genetic risk factors for periodontal diseases: is the climb worth the view? Perio 20002002 October;30:79-90.

Source of Support: Nil, Confilict of Interst: None Declared 